\title{
An Application of Artificial Intelligence for the Joint Estimation of Amplitude and Two-Dimensional Direction of Arrival of Far Field Sources Using 2-L-Shape Array
}

\author{
Fawad Zaman, ${ }^{1}$ Ijaz Mansoor Qureshi, ${ }^{1}$ Junaid Ali Khan, ${ }^{2}$ and Zafar Ullah Khan ${ }^{1}$ \\ ${ }^{1}$ Department of Electronic Engineering, Faculty of Engineering and Technology, \\ International Islamic University Sector H-10, Islamabad 44000, Pakistan \\ ${ }^{2}$ Department of Electrical Engineering, COMSATS Institute of Information Technology, Attock 43600, Pakistan
}

Correspondence should be addressed to Fawad Zaman; fawad.phdee31@iiu.edu.pk

Received 6 March 2013; Accepted 8 May 2013

Academic Editor: Juan Valenzuela-Valdes

Copyright (C) 2013 Fawad Zaman et al. This is an open access article distributed under the Creative Commons Attribution License, which permits unrestricted use, distribution, and reproduction in any medium, provided the original work is properly cited.

\begin{abstract}
An easy and efficient approach, based on artificial intelligence technique, is proposed to jointly estimate the amplitude, elevation, and azimuth angles of far field sources impinging on 2-L-shape array. In these proposed artificial intelligence techniques, the metaheuristics based on genetic algorithm and simulated annealing are used as global optimizers assisted with rapid local version of pattern search for optimization of the adaptive parameters. The performance metric is employed on a fitness evaluation function depending on mean square error which is optimum and requires single snapshot to converge. The proposed approaches are easy to understand, and simple to implement; the genetic algorithm specifically hybridized with pattern search generates fairly good results. The comparison of the given schemes is carried out with 1-L-shape array, as well as, with parallel-shape array and is found to be in good agreement in terms of accuracy, convergence rate, computational complexity, and mean square error. The effectiveness and efficiency of the given schemes are examined through Monte Carlo simulations and their inclusive statistical analysis.
\end{abstract}

\section{Introduction}

Two-dimensional direction of arrival (2D DOA) estimation for multiple signals received at sensors array is one of the indispensable steps for adaptive beamformer to maneuver the main beam in any preferred direction, while allocating nulls in the direction of unwanted signals $[1,2]$. From this perspective, 2D DOA estimation has direct applications in radar, sonar, and mobile communications [3]. The most common problem in 2D DOA estimation includes pair matching between elevation and azimuth angles, estimation failure and computational complexity. In the literature, many algorithms have already been discussed in the last two decades to estimate 2-D DOA [4-7], but they have the aforementioned problems. In [8], Wu et al. proposed an algorithm based on propagator method (PM) to overcome the computational load of [7], but it fails to avoid the pair matching problem and estimation failure problem in the range of 1.2217 (radians) to 1.5708 (radians). Moreover, the parallel-shape array is used in [8] which not only needs more sensors but also requires a large number of snapshots to achieve the goal (at least 200 snapshots per sensor are required). Besides that, [8] also failed to estimate the sources amplitudes which also an important parameter to be estimated.

In order to overcome these problems, we thank evolutionary computing techniques (ECTs) and L-shape arrays (1-L and 2-L shape). It is well acknowledged in the literature that ECTs such as genetic algorithm (GA), ant colony optimization (ACO), differential evolution (DE), and particle swarm optimization (PSO) have achieved great success in solving different optimization problems especially in the field of array signal processing $[9,10]$. These techniques have got attention among the researchers due to their ease in understanding, simplicity in implementation, and less probability to get stuck in the local minima. One of the other most significant aspects of ECT is that their accuracy and reliability increase more when hybridized with any other capable algorithm such as pattern search (PS), interior point algorithm (IPA), and active 


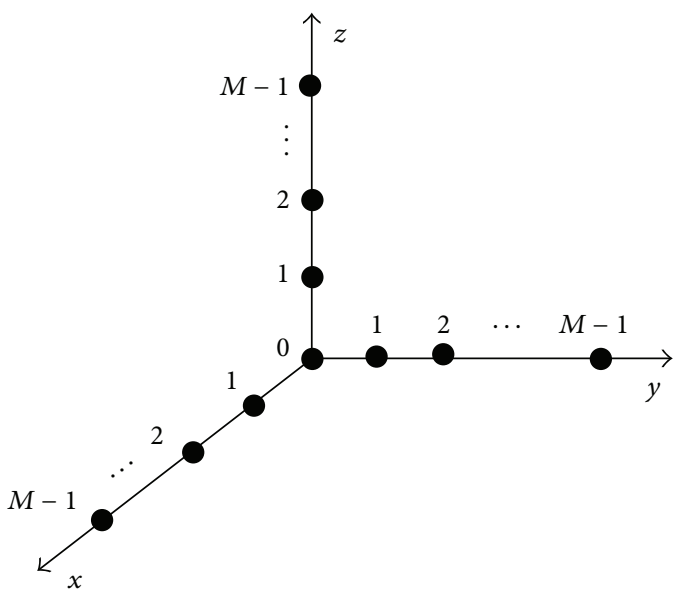

FIGURE 1: 2-L shape array consists of three subarrays.

set algorithm (ASA) [11-15]. In [13], GA is hybridized with IPA for 3D source localization of near field sources impinging on uniform linear array (ULA), and the hybrid GA-IPA approach has shown better performance as compared to GA and IPA alone. In [14], PSO is mixed with PS to estimate the DOA of far field sources, and again the hybrid PSO-PS technique produced better results as compared to PSO [9] and PS alone. GA is hybridized with PS to jointly estimate 3D parameters (amplitude, elevation angle, and azimuth angle) of far field sources arriving at 1-L shape array and again the results of hybrid GA-PS technique were remarkable as compared to GA and PS alone [15]. In [13-15], every time GA and PSO are treated as global optimizers, while PS and IPA are used as local search optimizers and mean square error (MSE) is used as a fitness function.

In this paper, an easy and efficient approach based on hybridized GA with PS (GA-PS) and hybrid simulated annealing (SA) technique with PS (SA-PS) are developed for joint estimation of amplitudes and 2-D DOA of far field sources impinging on 2-L-shape array. In these approaches GA and SA are exploited as global optimizers, while PS is incorporated for rapid local search to improve the results further. Mean square error (MSE) is used as an objective function based on an error between actual and estimated signals which is basically derived from maximum likelihood principle [13]. This fitness function needs single snapshot to achieve the results and avoids any uncertainty among the angles that are supplement to one another. The validity and effectiveness of the proposed approaches are tested on a large number of simulations which include different cases based on a different number of sources, a different number of sensors, computational complexity, and the proximity of the sources. Initially, the results of GA, PS, SA, SA-PS, and GA-PS are compared with each other for 2-L-shape array, and then, the entire results are compared with the results of 1-L-shape array [15]. Later on, the results are also compared with parallel shape array which uses PM [8].

Throughout the paper, the matrices are denoted by capital bold letters, while vectors are expressed by small bold letters, whereas " $T$ " denotes the transpose of vectors or matrices.
The left behind paper is organized as follows: the problem formulation is discussed in Section 2, whereas its proposed methodology is provided in Section 3. Section 4 demonstrates the comprehensive simulations along with the discussion of the results, while conclusion and future directions for the research are revealed in Section 5.

\section{Problem Formulation}

In this section, we addressed the unknown parameters of the received signals. Consider a 2 -L-shape array which consists of three uniform linear arrays (ULAs) placed along $x$-axis, $y$ axis, and $z$-axis. Each ULA contains $M-1$ elements with the same interelement spacing " $d$ ", while the reference sensor is common for all subarrays as shown in Figure 1 [16]. Consider $K$ narrow band sources impinging on the 2-L-shape array where $s_{i}, \theta_{i}$, and $\phi_{i}$ are the $i$ th source amplitude, elevation angle, and azimuth angle, respectively.

Output Vector along $z$-Axis Subarray. The subarray along $z$ axis is used to estimate the elevation angle. The signal received at $l$ th element from $K$ sources can be written as

$$
w_{z l}=\sum_{i=1}^{K} b_{z l}\left(\theta_{i}\right) s_{i}+\alpha_{z l},
$$

where $l=0,1,2, \ldots, M-1$.

In matrix-vector form, the output of entire subarray can be written as

$$
\mathbf{w}_{z}=\mathbf{B}_{z}(\theta) \mathbf{s}+\boldsymbol{\alpha}_{z}
$$

where $\mathbf{B}$ is called steering matrix, which contains steering vectors; that is,

$$
\mathbf{B}_{z}=\left[\mathbf{b}_{z 0}, \mathbf{b}_{z 1}, \ldots, \mathbf{b}_{z M-1}\right]^{T}
$$

where

$$
\begin{gathered}
\mathbf{b}_{z l}=\left[b_{z l}\left(\theta_{1}\right), b_{z l}\left(\theta_{2}\right), \ldots, b_{z l}\left(\theta_{k}\right)\right], \\
b_{z l}\left(\theta_{i}\right)=\exp \left(-j \psi_{l, i}\right), \quad \psi_{l, i}=\frac{2 \pi l d \cos \theta_{i}}{\lambda} \\
\text { for } i=1,2, \ldots, K .
\end{gathered}
$$

Similarly, in (2),

$$
\begin{gathered}
\mathbf{w}_{z}=\left[w_{z 0}, w_{z 1}, \ldots, w_{z M-1}\right]^{T}, \\
\mathbf{s}=\left[s_{1}, s_{2}, \ldots, s_{K}\right]^{T}, \\
\boldsymbol{\alpha}_{z}=\left[\alpha_{z 0}, \alpha_{z 1}, \ldots, \alpha_{z M-1}\right]^{T},
\end{gathered}
$$

where $\boldsymbol{\alpha}_{z}$ is called additive white Gaussian noise (AWGN) added at the output of each sensor placed in $z$-axis subarray.

Output Vector along $x$-Axis Subarray. The subarray along $x$ axis is used to estimate the $x$-component of azimuth angle and elevation angle. The output at $l$ th sensor placed in the $x$ axis subarray for $K$ sources can be written as

$$
w_{x l}=\sum_{i=1}^{K} b_{x l}\left(\theta_{i}, \phi_{i}\right) s_{i}+\alpha_{x l} .
$$


TABLE 1: Parameter settings for GA, SA, and PS.

\begin{tabular}{|c|c|c|c|c|c|}
\hline \multicolumn{2}{|l|}{ GA } & \multicolumn{2}{|c|}{ PS } & \multicolumn{2}{|c|}{ SA } \\
\hline Parameters & Settings & Parameters & Setting & Parameters & Setting \\
\hline Population size & 240 & Poll method & GPS positive basis $2 \mathrm{~N}$ & Annealing function & Fast \\
\hline No. of generations & 1000 & Polling order & Consecutive & Reannealing interval & 100 \\
\hline Migration direction & Both ways & Maximum iteration & 800 & $\begin{array}{l}\text { Temperature update } \\
\text { function }\end{array}$ & $\begin{array}{c}\text { Exponential } \\
\text { temperature update }\end{array}$ \\
\hline Crossover fraction & 0.2 & Function evaluation & 16000 & Initial temperature & 100 \\
\hline Crossover & Heuristic & Mesh size & 01 & Data type & Custom \\
\hline Function tolerance & $10-12$ & Expansion factor & 2.0 & Function tolerance & $10-12$ \\
\hline Initial range & {$[0-1]$} & Contraction factor & 0.5 & Max iteration & 2000 \\
\hline Scaling function & Rank & Penalty factor & 100 & Max function evaluations & $\begin{array}{c}3000 * \text { number of } \\
\text { variables }\end{array}$ \\
\hline Selection & $\begin{array}{l}\text { Stochastic } \\
\text { uniform }\end{array}$ & Bind tolerance & $10-03$ & $\begin{array}{l}\text { Temperature update } \\
\text { function }\end{array}$ & $\begin{array}{c}\text { Exponential } \\
\text { temperature update }\end{array}$ \\
\hline Elite count & 2 & Mesh tolerance & $10-06$ & & \\
\hline Mutation function & $\begin{array}{l}\text { Adaptive } \\
\text { feasible }\end{array}$ & $\mathrm{X}$ tolerance & $10-06$ & $\begin{array}{l}\text { Hybrid function call } \\
\text { interval }\end{array}$ & End \\
\hline
\end{tabular}

In vector-matrix form, it can be written as

$$
\mathbf{w}_{x}=\mathbf{B}_{x}(\theta, \phi) \mathbf{s}+\boldsymbol{\alpha}_{x},
$$

where

$$
\begin{gathered}
\mathbf{B}_{x}=\left[\mathbf{b}_{x 0}, \mathbf{b}_{x 1}, \ldots, \mathbf{b}_{x M-1}\right]^{T}, \\
\mathbf{b}_{x l}=\left[b_{x l}\left(\theta_{1}, \phi_{1}\right), b_{x l}\left(\theta_{2}, \phi_{2}\right), \ldots, b_{x l}\left(\theta_{k}, \phi_{k}\right)\right], \\
b_{x l}\left(\theta_{i}, \phi_{i}\right)=\exp \left(-j \psi_{l, i}\right), \quad \psi_{l, i}=\frac{2 \pi l d \sin \theta_{i} \cos \phi_{i}}{\lambda} .
\end{gathered}
$$

Similarly,

$$
\begin{aligned}
\mathbf{w}_{x} & =\left[w_{x 0}, w_{x 1}, \ldots, w_{x M-1}\right]^{T}, \\
\boldsymbol{\alpha}_{x} & =\left[\alpha_{x 0}, \alpha_{x 1}, \ldots, \alpha_{x M-1}\right]^{T},
\end{aligned}
$$

where $\boldsymbol{\alpha}_{x}$ is AWGN added at the output of each sensor in $x$ axis subarray.

Output Vector along $y$-Axis Subarray. In the same manner, the $y$-axis subarray is used to estimate the $y$-component of elevation and azimuth angle. The output at $l$ th sensor in this subarray is given as follows:

$$
\begin{gathered}
w_{y l}=\sum_{i=1}^{K} b_{y l}\left(\theta_{i}, \phi_{i}\right) s_{i}+\alpha_{y l}, \\
\mathbf{w}_{y}=\mathbf{B}_{y}(\theta, \phi) \mathbf{s}+\boldsymbol{\alpha}_{y},
\end{gathered}
$$

where

$$
\mathbf{B}_{y}=\left[\mathbf{b}_{y 0}, \mathbf{b}_{y 1}, \ldots, \mathbf{b}_{y M-1}\right]^{T},
$$

where

$$
\begin{gathered}
\mathbf{b}_{y l}=\left[b_{y l}\left(\theta_{1}, \phi_{1}\right), b_{y l}\left(\theta_{2}, \phi_{2}\right), \ldots, b_{y l}\left(\theta_{k}, \phi_{k}\right)\right], \\
b_{y l}\left(\theta_{i}, \phi_{i}\right)=\exp \left(-j \psi_{l, i}\right), \quad \psi_{l, i}=\frac{2 \pi l d \sin \theta_{i} \sin \phi_{i}}{\lambda} .
\end{gathered}
$$

Similarly,

$$
\begin{aligned}
\mathbf{w}_{y} & =\left[w_{y 0}, w_{y 1}, \ldots, w_{y M-1}\right]^{T}, \\
\boldsymbol{\alpha}_{y} & =\left[\alpha_{y 0}, \alpha_{y 1}, \ldots, \alpha_{y M-1}\right]^{T},
\end{aligned}
$$

where $\boldsymbol{\alpha}_{y}$ is AWGN added at the output of each sensor in $y$ axis subarray. Here, the problem in hand is very clear which is to estimate the unknown parameters, that is, amplitudes and 2D-DOA (elevation, azimuth) angles for $K$ sources impinging from far field on 2-L-shape array.

\section{Proposed Techniques}

In this section, a brief introduction, parameter setting, and flow diagram are given for GA, PS, SA, GA-PS, and SA-PS.

Simulated Annealing. Simulated annealing (SA) technique is used for both discrete and continuous optimization problems. SA works on those problems, which have controlled heating and cooling properties as evident from its name "annealing", which means "To heat and then cool." Due to easiness in implementation and the capability to avoid getting stuck in local minima, SA is a well-liked technique over the last few decades and has been successfully used in wide range of engineering problems [17]. In this paper, an MATLAB "built-in optimization tool box" is used for which the parameter setting is shown in Table 1 . The best individual result of SA is further passed through PS for further tuning.

Pattern Search. Pattern search (PS) was introduced by Hookes and Jeeves in 1961 which does not require the gradient or derivative of the problem and can be used for both local and global optimization problems. Basically, PS works on mesh which is defined according to some specific rules. If no improvement in cost function is achieved at the mesh points of current iteration, then the mesh is polished and the process 


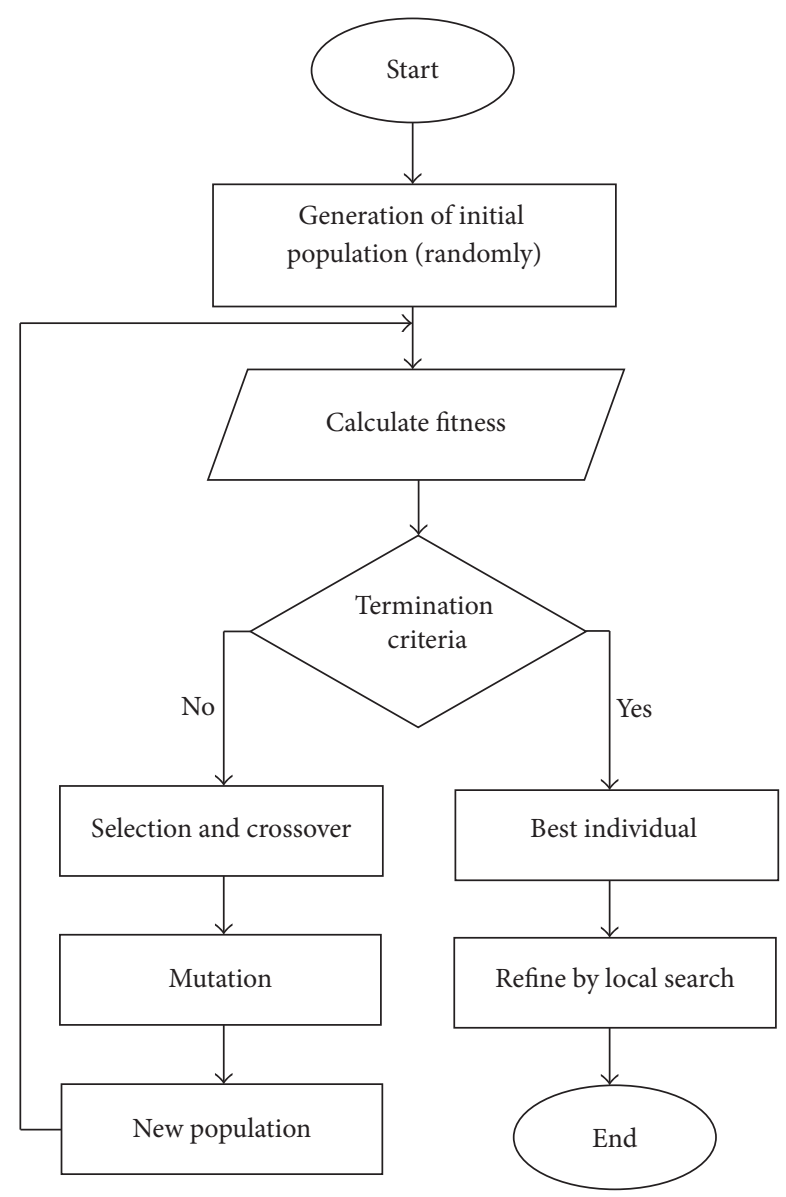

FIGURE 2: Generic structure of the hybrid intelligent approach.

is repeated. It has already got application almost in every field such as signal processing and soft computing [18].

Genetic Algorithm. GA is basically different from previously discussed algorithm (SA and PS) and is applicable to a wide range of optimization problems. GA is a more prominent and proficient algorithm than any other evolutionary computing technique due to its ease in conception, ease in implementation, and more importantly less probability to get stuck in the presence of local optima. GA is being successfully applied to a wide range of problems having application in commerce to the leading scientific research $[19,20]$. The structure of GA exploited for the joint estimation is given in Figure 2.

The general settings consisting of population size, number of generations of the algorithm, function evaluations, and stoppage criteria are defined in Table 1, along with some specific parameter setting/values of the three algorithms based on GA, PS, and SA.

The logical steps for GA and GA-PS in the form of pseudo code are given in the following steps.

Step 1 (initialization). Generate randomly $Q$ number of chromosomes (particles) where the length of each chromosome is $3 \times K$. In each chromosome, the first $K$ genes represent amplitudes, the next $K$ genes contain the elevation angles, while the last $K$ genes represent the azimuth angles. Mathematically, the $q$ th chromosome can be written as

$$
\mathbf{d}_{q}=\left[s_{q, 1}, s_{q, 2}, \ldots, s_{q, K}, \theta_{q, K+1}, \ldots, \theta_{q, 2 K}, \phi_{q, 2 K+1}, \ldots, \phi_{q, 3 K}\right] .
$$

Here, $s_{q j} \in R: L_{a} \leq s_{q j} \leq U_{a}$ where $L_{a}$ and $U_{a}$ are the lower and upper bounds of signal amplitudes $\forall q=1,2, \ldots, Q$ and $j=1,2, \ldots, K$. In the same way, the elevation and azimuth angles are

$$
\begin{array}{cc} 
& \theta_{q j} \in R: 0 \leq \theta_{q j} \leq \pi, \\
\forall q=1,2, \ldots, Q, \quad j=K+1, K+2, \ldots, 2 K, \\
\\
\phi_{q j} \in R: 0 \leq \phi_{q j} \leq 2 \pi, \\
\forall q=1,2, \ldots, Q, \quad j=2 K+1,2 K+2, \ldots, 3 K .
\end{array}
$$

Step 2 (objective function). MSE is used as an objective evaluation function, which is derived from MLP [13]. For $q$ th chromosome, it can be written as

$$
E(q)=E_{x}(q)+E_{y}(q)+E_{z}(q),
$$

where

$$
\begin{aligned}
& E_{x}(q)=\frac{1}{(M-1)} \sum_{x=1}^{M-1}\left|w_{x}-\widehat{w}_{x}^{q}\right|^{2}, \\
& E_{y}(q)=\frac{1}{(M-1)} \sum_{y=1}^{M-1}\left|w_{y}-\widehat{w}_{y}^{q}\right|^{2}, \\
& E_{z}(q)=\frac{1}{(M-1)} \sum_{z=1}^{M-1}\left|w_{z}-\widehat{w}_{z}^{q}\right|^{2} .
\end{aligned}
$$

$w_{z}, w_{x}$, and $w_{y}$ are defined in (1), (3), and (7), respectively, while $\widehat{w}_{x}^{q}, \widehat{w}_{y}^{q}$, and $\widehat{w}_{z}^{q}$ at $l$ th element of each subarray for $q$ th chromosome are given as

$$
\begin{gathered}
\widehat{w}_{x l}^{q}=\sum_{i=1}^{K} \exp \left[-j \pi l \sin \left(d_{q, K+i}\right) \cos \left(d_{q, 2 K+i}\right)\right] d_{q, i}, \\
\widehat{w}_{y l}^{q}=\sum_{i=1}^{K} \exp \left[-j \pi l \sin \left(d_{q, K+i}\right) \sin \left(d_{q, 2 K+i}\right)\right] d_{q, i}, \\
\widehat{w}_{z l}^{q}=\sum_{i=1}^{K} \exp \left[-j \pi l \cos \left(d_{q, K+i}\right)\right] d_{q, i},
\end{gathered}
$$

where $d_{q}$ is defined in (14).

Step 3 (termination criteria). The termination criteria depending on the following conditions are achieved.

(a) If the objective function value is achieved, which is predefined, that is, $\varepsilon \leq 10^{-12}$.

(b) The total number of iterations is completed.

(c) The Tol Fun exceeded.

(d) Tol Con occurred. 
TABLE 2: Estimation accuracy of 2-L-shape array for 2 sources.

\begin{tabular}{lcccccc}
\hline Scheme & $s_{1}$ & $s_{2}$ & $\theta_{1}(\mathrm{rad})$ & $\theta_{2}(\mathrm{rad})$ & $\phi_{1}(\mathrm{rad})$ & 1.2217 \\
\hline Desired values & 1.0000 & 2.0000 & 0.5236 & 0.8727 & 0.8731 & 1.2221 \\
GA & 1.0003 & 2.0003 & 0.5240 & 0.8748 & 1.2239 & 1.9199 \\
PS & 1.0020 & 2.0021 & 0.5259 & 0.8889 & 1.2361 & 1.92220 \\
SA & 1.0977 & 2.0178 & 0.5385 & 0.8770 & 1.2260 \\
SA-PS & 1.0047 & 2.0047 & 0.5278 & 0.8726 & 1.2216 \\
GA-PS & 1.0000 & 2.0000 & 0.5235 & 1.9242 \\
\hline
\end{tabular}

TABLE 3: Estimation accuracy of 1-L-type array for 2 sources [15].

\begin{tabular}{|c|c|c|c|c|c|c|}
\hline Scheme & $s_{1}$ & $s_{2}$ & $\theta_{1}(\mathrm{rad})$ & $\theta_{2}(\mathrm{rad})$ & $\phi_{1}(\mathrm{rad})$ & $\phi_{2}(\mathrm{rad})$ \\
\hline Desired values & 1.0000 & 2.0000 & 0.5236 & 0.8727 & 1.2217 & 1.9199 \\
\hline GA & 1.0008 & 2.0008 & 0.5243 & 0.8735 & 1.2225 & 1.9207 \\
\hline PS & 1.0032 & 2.0033 & 0.5268 & 0.8759 & 1.2249 & 1.9231 \\
\hline SA & 1.0196 & 2.0195 & 0.5432 & 0.8923 & 1.2413 & 1.9395 \\
\hline SA-PS & 1.0063 & 2.0063 & 0.5299 & 0.8790 & 1.2281 & 1.9263 \\
\hline GA-PS & 1.0003 & 2.0002 & 0.5233 & 0.8723 & 1.2220 & 1.9195 \\
\hline
\end{tabular}

Step 4 (reproduction). New population is generated by using the operators of crossover, elitism, and mutation selection as provided in Table 1.

Step 5 (hybridization). In this step, the finest results got through GA are further given to PS for more improvement. The setting used for PS is also provided in Table 1.

Step 6 (storage). Store global best of the current iteration which will be used for comparison and better statistical analysis, and repeat Steps 2 to 5 for enough numbers of independent runs.

\section{Results and Discussions}

This section is broadly divided into two parts. In first part of simulations, GA, PS, SA, GA-PS, and SA-PS are examined for 2-L-shape array in terms of estimation accuracy, convergence rate, and at the same time, the results are compared with 1L-shape array [15]. In the second part, the performance of the best scheme among the above-mentioned five schemes for 2-L-shape array is not only compared with 1-L-shape array but also with parallel-shape array [8]. Throughout the simulations, the distance " $d$ " between two consecutive sensors in each subarray is kept $\lambda / 2$. All the values of the DOA (elevation and azimuth angle) of sources are taken in radian (rad), and all the results for 2-L-shape array are carried out for threshold value of MSE $10^{-2}$. The MATLAB built-in "Optimization Tool Box" is used for GA, SA, and PS for which the settings are provided in Table 1 . As in [15], no noise is added to the system for estimation accuracy and convergence rate. All the results are comprehensively examined for 100 independent runs.

Case 1. In this case, the estimation accuracy of GA, PS, SA, GA-PS, and SA-PS is discussed for two sources imping- ing on 2-L-type array. For better comparison and analysis, all the values of amplitudes and DOA are the same as in [15] for 1-L-shape array, so the amplitudes and DOA values are $\left\{s_{1}, s_{2}, \theta_{1}, \theta_{2}, \phi_{1}, \phi_{2}\right\}=\{1,2,0.5236(\mathrm{rad}), 0.8727(\mathrm{rad})$, $1.2217(\mathrm{rad}), 1.9199(\mathrm{rad})\}$ where $s_{1}, \theta_{1}$, and $\phi_{1}$ represent the amplitudes, elevation angle, and azimuth angles of first source and so on. The 2-L-shape array is composed of 4 sensors in which 1 sensor is placed along each subarray, while the reference sensor is common for them. As provided in Table 2, all the five mentioned techniques are quite capable of producing fairly good estimate of the actual values. However, among them, the hybrid approach GA-PS produced better results as compared to the other techniques used. The second and third best results are provided by GA and PS, respective ly. The results of SA are also improved when hybridized with PS.

The results of 1-L-shape array for 2 sources are provided in Table 3 which requires 7 sensors [15]. The 1-L-shape array in [15] also uses the same techniques. One can clearly deduce from the comparison of Tables 2 and 3 that 2-L-shape array produced more accurate results as compared to 1-L-shape array with less number of sensors.

Case 2. In this case, the estimation accuracy of all the abovementioned five techniques is discussed for three sources impinging on 2-L-shape array. This time the 2-L-shape array consists of 7 sensors; that is, 2 sensors are placed along each subarray, while the reference sensor is common for them. For better comparison with 1-L-type array, the same values of amplitudes and DOA are used as in [15], which are provided in Table 4. In this case, few local minima are observed due to which the performance of all five techniques especially SA, SA-PS, and PS is despoiled. However, again the hybrid GAPS shows excellence in accuracy even in the presence of local minima. The second best result is shown by GA alone.

Now compare the results with 1-L-type array as provided in Table 5 which requires thirteen sensors [15]. Now, one can observe that 2-L-shape array not only requires less number of 
TABLE 4: Estimation accuracy of 2-L-type array for 3 sources.

\begin{tabular}{lccccccccc}
\hline Scheme & $s_{1}$ & $s_{2}$ & $s_{3}$ & $\theta_{1}(\mathrm{rad})$ & $\theta_{2}(\mathrm{rad})$ & $\theta_{3}(\mathrm{rad})$ & $\phi_{1}(\mathrm{rad})$ & $\phi_{2}(\mathrm{rad})$ & $\phi_{3}(\mathrm{rad})$ \\
\hline Desired values & 1.0000 & 2.0000 & 3.0000 & 0.1745 & 0.8727 & 1.3090 & 0.5236 & 1.9199 & 2.4435 \\
GA & 1.0043 & 2.0043 & 3.0042 & 0.1788 & 0.8769 & 1.3132 & 0.5278 & 1.9243 & 2.4478 \\
PS & 1.0189 & 2.0190 & 3.0189 & 0.1934 & 0.9005 & 0.8917 & 0.5427 & 1.9389 & 2.4624 \\
SA & 1.0509 & 2.0508 & 3.0509 & 0.2254 & 0.9237 & 1.3598 & 0.5744 & 1.9708 & 2.4944 \\
SA-PS & 1.0342 & 2.0342 & 3.0343 & 0.2087 & 0.9069 & 1.3432 & 0.5581 & 1.9541 & 2.4778 \\
GA-PS & 1.0003 & 2.0004 & 3.0003 & 0.1748 & 0.8730 & 1.3094 & 0.5240 & 1.9202 & 2.4439 \\
\hline
\end{tabular}

TABLE 5: Performance of 1-L-type array for 3 sources [15].

\begin{tabular}{|c|c|c|c|c|c|c|c|c|c|}
\hline Scheme & $s_{1}$ & $s_{2}$ & $s_{3}$ & $\theta_{1}(\mathrm{rad})$ & $\theta_{2}(\mathrm{rad})$ & $\theta_{3}(\mathrm{rad})$ & $\phi_{1}(\mathrm{rad})$ & $\phi_{2}(\mathrm{rad})$ & $\phi_{3}(\mathrm{rad})$ \\
\hline Desired values & 1.0000 & 2.0000 & 3.0000 & 0.1745 & 0.8727 & 1.3090 & 0.5236 & 1.9199 & 2.4435 \\
\hline GA & 1.0073 & 2.0073 & 3.0073 & 0.1818 & 0.8800 & 1.3163 & 0.5309 & 1.9272 & 2.4508 \\
\hline PS & 1.0278 & 2.0277 & 3.0277 & 0.2023 & 0.9005 & 1.3368 & 0.5514 & 1.9477 & 2.4713 \\
\hline SA & 1.0610 & 2.0611 & 3.0610 & 0.2355 & 0.9337 & 1.3700 & 0.5846 & 1.9809 & 2.5045 \\
\hline SA-PS & 1.0432 & 2.0432 & 3.0432 & 0.2177 & 0.9159 & 1.3522 & 0.5668 & 1.9631 & 2.4867 \\
\hline GA-PS & 1.0011 & 2.0011 & 3.0011 & 0.1756 & 0.8738 & 1.3101 & 0.5247 & 1.9210 & 2.4446 \\
\hline
\end{tabular}

sensors but also produces more accurate results as compared to 1-L-shape array.

Case 3. In this case, the estimation accuracy is examined for 4 -sources. The 2 -L-shape array consists of 10 sensors; that is, 3 elements are placed along each subarray, while the reference element is common for them. As provided in Table 6, again the hybrid GA-PS leads the edge over the remaining four techniques in terms of estimation accuracy. The results for 1L-shape array for the same schemes and the same number of sources are provided in Table 7 which needs fifteen sensors [15]. One can again make out the advantages of 2-L-shape array over 1-L-type array in terms of accuracy and the number of sensors required.

Case 4. In this case, the convergence rate is evaluated for 2-Lshape array against a different number of sources in the presence of $10 \mathrm{~dB}$ noise. As shown in Figure 3, the convergence rates of all schemes are degraded with the increase of sources. However, one can notice that the convergence rate of GA is remarkable in case of hybridization with PS. The second best convergence is produced by GA itself alone.

Now, by comparing with 1-L-shape array whose convergence rate is shown in Figure 4, one can conclude that all the five schemes have better convergence rate for 2-L-shape array as compared to 1-L-shape array.

Case 5. In this case, we checked the validation of all techniques for more practical scenario; that is, we considered DOA on the reference axis of L-shape arrays. For this, the 2$\mathrm{L}$-shape array is composed of 12 sensors where the amplitudes and DOA of the four sources are

$$
\begin{gathered}
\left\{s_{1}=2, \theta_{1}=1.5702(\mathrm{rad}), \phi_{1}=0(\mathrm{rad})\right\}, \\
\left\{s_{2}=5, \theta_{2}=1.2217(\mathrm{rad}), \phi_{2}=6.2832(\mathrm{rad})\right\},
\end{gathered}
$$

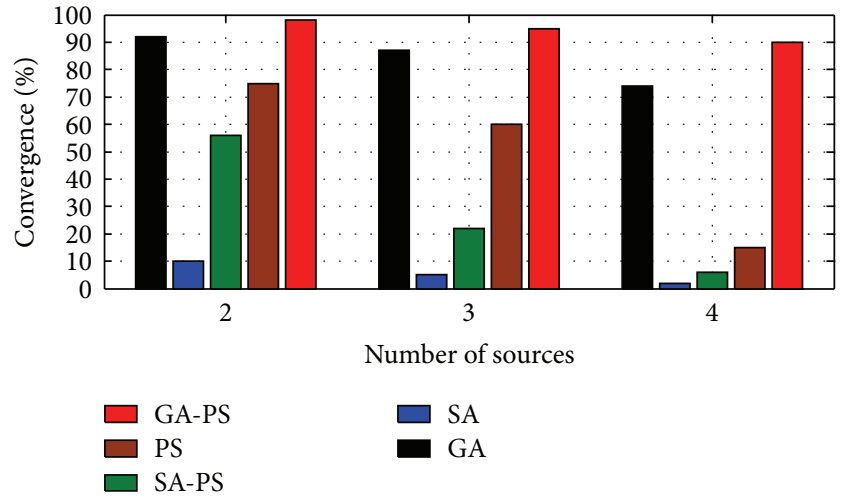

FIGURE 3: Convergence rate versus number of sources for 2-L-shape array at $10 \mathrm{~dB}$ noise.

$$
\begin{gathered}
\left\{s_{3}=1, \theta_{3}=0(\mathrm{rad}), \phi_{3}=4.7124(\mathrm{rad})\right\}, \\
\left\{s_{4}=4, \theta_{4}=1.0472(\mathrm{rad}), \phi_{4}=1.5702(\mathrm{rad})\right\} .
\end{gathered}
$$

As provided in Table 8, an obvious degradation can be observed in the estimation accuracy of each scheme for all the elevation angles taken near the reference axis, while for azimuth angle near the reference axis, the accuracy is slightly degraded. However, among all of them the GA-PS technique is less degraded as compared to the others.

Now, if we check the same DOA using 1-L-shape array, then one can see in Table 9 that the estimation accuracy of all schemes is degraded more as compared to 2-L-shape array.

Up till now, the performance of GA, PS, SA, SA-PS, and GA-PS is discussed for both 1-L- and 2-L-type arrays, and it has been shown through various cases that GA-PS produced fairly good results for both arrays. So, from now onwards, our focus will be limited to the GA-PS technique only. In the upcoming second part of the simulations, we will compare 
TABLE 6: Performance of 2-L-type array for 4 sources.

\begin{tabular}{lcccccccccccc}
\hline Scheme & $s_{1}$ & $s_{2}$ & $s_{3}$ & $s_{4}$ & $\theta_{1}(\mathrm{rad})$ & $\theta_{2}(\mathrm{rad})$ & $\theta_{3}(\mathrm{rad})$ & $\theta_{4}(\mathrm{rad})$ & $\phi_{1}(\mathrm{rad})$ & $\phi_{2}(\mathrm{rad})$ & $\phi_{3}(\mathrm{rad})$ & $\phi_{4}(\mathrm{rad})$ \\
\hline Desired & 1.0000 & 2.0000 & 3.0000 & 4.0000 & 0.2618 & 0.6109 & 1.0472 & 1.4835 & 1.6581 & 2.1817 & 2.7925 & 3.4034 \\
GA & 1.0102 & 2.0103 & 3.0103 & 4.0102 & 0.2721 & 0.6212 & 1.0576 & 1.4937 & 1.6683 & 2.1920 & 2.8028 & 3.4137 \\
PS & 1.0313 & 2.0312 & 3.0313 & 4.0314 & 0.2932 & 0.6423 & 1.0787 & 1.5148 & 1.6895 & 2.2132 & 2.8239 & 3.4348 \\
GA-PS & 1.0040 & 2.0042 & 3.0043 & 4.0040 & 0.2659 & 0.6151 & 1.0514 & 1.4877 & 1.6624 & 2.1858 & 2.7966 & 3.4077 \\
SA & 1.1011 & 2.1010 & 3.1012 & 4.1010 & 0.3628 & 0.7122 & 1.1483 & 1.5846 & 1.7594 & 2.2829 & 2.8937 & 3.5044 \\
SA-PS & 1.0787 & 2.0786 & 3.0785 & 4.0787 & 0.3405 & 0.6895 & 1.1260 & 1.5624 & 1.7369 & 2.2606 & 2.8713 & 3.4821 \\
\hline
\end{tabular}

TABLE 7: Performance of 1-L-type array for 4 sources [15].

\begin{tabular}{lcccccccccccc}
\hline Scheme & $s_{1}$ & $s_{2}$ & $s_{3}$ & $s_{4}$ & $\theta_{1}(\mathrm{rad})$ & $\theta_{2}(\mathrm{rad})$ & $\theta_{3}(\mathrm{rad})$ & $\theta_{4}(\mathrm{rad})$ & $\phi_{1}(\mathrm{rad})$ & $\phi_{2}(\mathrm{rad})$ & $\phi_{3}(\mathrm{rad})$ & $\phi_{4}(\mathrm{rad})$ \\
\hline Desired & 1.0000 & 2.0000 & 3.0000 & 4.0000 & 0.2618 & 0.6109 & 1.0472 & 1.4835 & 1.6581 & 2.1817 & 2.7925 & 3.4034 \\
GA & 1.0163 & 2.0163 & 3.0162 & 4.0163 & 0.2781 & 0.6272 & 1.0635 & 1.4998 & 1.6744 & 2.1980 & 2.8088 & 3.4197 \\
PS & 1.0425 & 2.0425 & 3.0426 & 4.0426 & 0.3043 & 0.6534 & 1.0897 & 1.5260 & 1.7006 & 2.2242 & 2.8350 & 3.4468 \\
GA-PS & 1.0083 & 2.0083 & 3.0083 & 4.0083 & 0.2701 & 0.6192 & 1.0555 & 1.4918 & 1.6664 & 2.1900 & 2.8008 & 3.4117 \\
SA & 1.1263 & 2.1263 & 3.1263 & 4.1164 & 0.3881 & 0.7372 & 1.1735 & 1.6098 & 1.7844 & 2.3080 & 2.9188 & 3.5306 \\
SA-PS & 1.0932 & 2.0932 & 3.0932 & 4.0932 & 0.3550 & 0.7041 & 1.1404 & 1.5767 & 1.7513 & 2.2749 & 2.8857 & 3.4966 \\
\hline
\end{tabular}

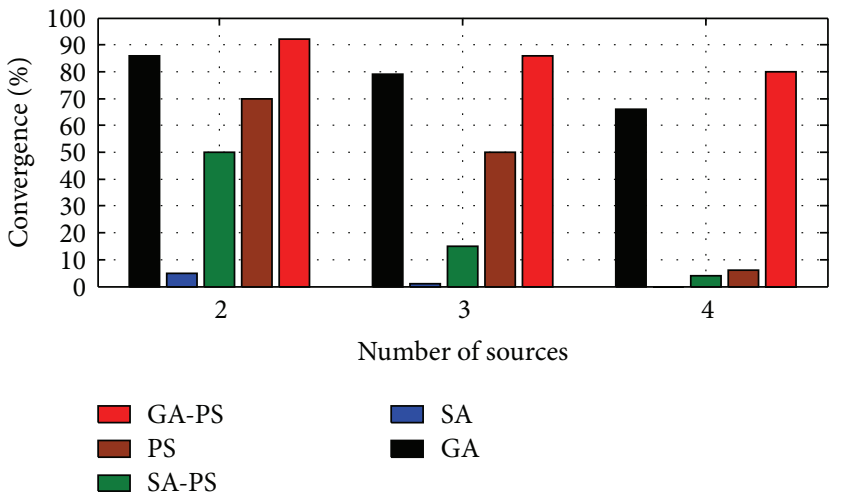

FIGURE 4: Convergence rate versus number of sources for 1-L-shape array at $10 \mathrm{~dB}$ noise.

the GA-PS technique used for 2-L-shape array not only with GA-PS technique used for 1-L-shape array but also with PM which utilizes parallel-shape array [8].

Case 6. In this case, the estimation accuracy in terms of MSE is discussed for GA-PS technique against the increasing number of sources. For this, the 2-L-shape array is composed of 13 sensors, while the 1-L-shape array is consisting of 19 sensors. As shown in Figure 5, the MSE is degraded for both L-shape arrays against the increasing number of sources. However, the MSE of GA-PS for 2-L-shape array is better than 1-L-shape array.

Case 7. In this case, Tables 10,11 , and 12 list the variances, means, and standard deviations for parallel-shape array with PM method and L-shape arrays with hybrid GA-PS approach. For this, the elevation angle varies between 1.2217 (rad) and 1.5708 (rad) for a fixed azimuth angle of 0.6109 (rad) in the presence of $10 \mathrm{~dB}$ noise.

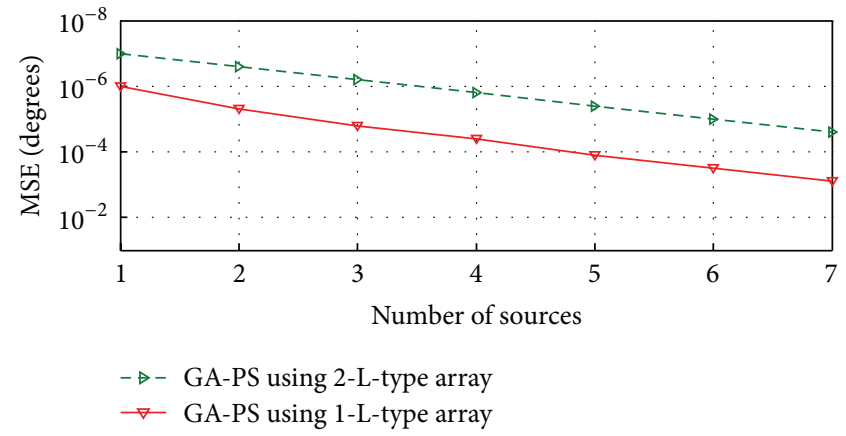

FIGURE 5: MSE versus number of sources at $10 \mathrm{~dB}$ noise.

The performance of PM method with parallel-shape array is worse for this range of elevation angle, while the 2-L-shape array with GA-PS technique produced accurate estimation. The second best result is produced by GA-PS for 1-L-shape array. This range of elevation angles is very important for mobile communication, and hence, 2-L-shape array with GA-PS is a good choice to be used. Moreover, the range of elevation and azimuth angles for 2-L-shape array is $(0, \pi)$ and $(0,2 \pi)$, respectively, while the parallel-shape array and $1-\mathrm{L}-$ shape array have the range of elevation, and azimuth angles are $(0, \pi / 2)$ and $(0,2 \pi)$, respectively, so both arrays cannot be operated for elevation angles beyond $\pi / 2$. For more details, the readers are encouraged to see [21].

Case 8. In this case, we discussed the computational complexity of GA-PS used for L-shape arrays and PM with parallel-shape array. The $\mathrm{PM}$ required total $\mathrm{O}(3 \times M \times T \times K)$ computations where $M$ is the total number of sensors, $K$ is the total number of sources, and $T$ is the number of snapshots which is 200 [8]. On the other hand, the major computations involved in GA-PS with 2-L-shape array include the total number of multiplication in fitness function $O\left(Q^{2}(3+\right.$ 
TABLE 8: Performance of 2-L-type array for sources located on reference axis.

\begin{tabular}{lcccccccccccc}
\hline Scheme & $s_{1}$ & $s_{2}$ & $s_{3}$ & $s_{4}$ & $\theta_{1}(\mathrm{rad})$ & $\theta_{2}(\mathrm{rad})$ & $\theta_{3}(\mathrm{rad})$ & $\theta_{4}(\mathrm{rad})$ & $\phi_{1}(\mathrm{rad})$ & $\phi_{2}(\mathrm{rad})$ & $\phi_{3}(\mathrm{rad})$ & $\phi_{4}(\mathrm{rad})$ \\
\hline Desired & 2.0000 & 5.0000 & 1.0000 & 4.0000 & 1.5702 & 1.2217 & 0.0000 & 1.0472 & 0.0000 & 6.2832 & 4.7124 & 1.5702 \\
GA & 1.9897 & 5.0105 & 0.9897 & 4.0103 & 1.6633 & 1.2321 & 0.0838 & 1.0573 & 0.0192 & 6.3041 & 4.6914 & 1.5900 \\
PS & 2.0315 & 4.9688 & 0.9687 & 3.9687 & 1.7139 & 1.2485 & 0.1623 & 1.0785 & 0.0314 & 6.2500 & 4.7473 & 1.6090 \\
GA-PS & 2.0039 & 4.9958 & 1.0044 & 4.0041 & 1.6197 & 1.2259 & 0.0524 & 1.0514 & 0.0105 & 6.2919 & 4.7211 & 1.5830 \\
SA & 1.8989 & 5.1011 & 1.1010 & 3.8989 & 1.3439 & 1.3231 & 0.2234 & 1.1483 & 0.0489 & 6.3303 & 4.7613 & 1.5237 \\
SA-PS & 1.9213 & 4.9214 & 1.0783 & 3.9213 & 1.7453 & 1.3001 & 0.1955 & 1.1261 & 0.0401 & 6.3216 & 4.7490 & 1.5341 \\
\hline
\end{tabular}

TABLE 9: Performance of 1-L-type array for sources located on reference axis [15].

\begin{tabular}{lcccccccccccc}
\hline Scheme & $s_{1}$ & $s_{2}$ & $s_{3}$ & $s_{4}$ & $\theta_{1}(\mathrm{rad})$ & $\theta_{2}(\mathrm{rad})$ & $\theta_{3}(\mathrm{rad})$ & $\theta_{4}(\mathrm{rad})$ & $\phi_{1}(\mathrm{rad})$ & $\phi_{2}(\mathrm{rad})$ & $\phi_{3}(\mathrm{rad})$ & $\phi_{4}(\mathrm{rad})$ \\
\hline Desired & 2.0000 & 5.0000 & 1.0000 & 4.0000 & 1.5702 & 1.2217 & 0.0000 & 1.0472 & 0.0000 & 6.2832 & 4.7124 & 1.5702 \\
GA & 2.0163 & 4.9837 & 1.0162 & 3.9837 & 1.7069 & 1.2381 & 0.1396 & 1.0635 & 0.0357 & 6.3190 & 4.7483 & 1.6059 \\
PS & 2.0425 & 4.9575 & 1.0426 & 4.0426 & 1.7698 & 1.2643 & 0.1991 & 1.0897 & 0.0598 & 6.3430 & 4.7721 & 1.6299 \\
GA-PS & 1.9917 & 5.0083 & 1.0083 & 4.0083 & 1.4661 & 1.2300 & 0.1065 & 1.0555 & 0.0375 & 6.2798 & 4.7301 & 1.5910 \\
SA & 2.1263 & 5.1263 & 0.8737 & 3.8836 & 1.8500 & 1.3480 & 0.2741 & 1.1735 & 0.0792 & 6.3467 & 4.7741 & 1.5067 \\
SA-PS & 1.9068 & 5.0932 & 0.9068 & 4.0932 & 1.3334 & 1.3150 & 0.2287 & 1.1405 & 0.0668 & 6.3328 & 4.7563 & 1.265 \\
\hline
\end{tabular}

TABLE 10: Means, variances, and standard deviations at $10 \mathrm{~dB}$ noise for different elevation angles and fixed azimuth angle by using PM with parallel-shape array [8].

\begin{tabular}{lccc}
\hline $\begin{array}{l}\theta \text { in radians for } \\
\phi=0.6109(\mathrm{rad})\end{array}$ & $\begin{array}{c}\text { Mean of } \theta \\
(\mathrm{rad})\end{array}$ & $\begin{array}{c}\text { Variance of } \theta \\
(\mathrm{rad})\end{array}$ & $\begin{array}{c}\text { Standard deviation } \\
\text { of } \theta(\mathrm{rad})\end{array}$ \\
\hline 1.2392 & 1.2227 & 0.0123 & 0.0146 \\
1.2915 & 1.2728 & 0.0171 & 0.0167 \\
1.3439 & 1.3207 & 0.0228 & 0.0200 \\
1.3963 & 1.3696 & 0.0403 & 0.0265 \\
1.4486 & 1.4155 & 0.0822 & 0.0379 \\
1.5010 & 1.4552 & 0.1250 & 0.0467 \\
1.5533 & 1.4784 & 0.1631 & 0.0534 \\
\hline
\end{tabular}

TABLE 11: Means, variances, and standard deviations at $10 \mathrm{~dB}$ noise for different elevation angles and fixed azimuth angle by using GAPS with 1-L-shape array [15].

\begin{tabular}{lccc}
\hline $\begin{array}{l}\theta \text { in radians for } \\
\phi=0.6109(\mathrm{rad})\end{array}$ & $\begin{array}{c}\text { Mean of } \theta \\
(\mathrm{rad})\end{array}$ & $\begin{array}{c}\text { Variance of } \theta \\
(\mathrm{rad})\end{array}$ & $\begin{array}{c}\text { Standard deviation } \\
\text { of } \theta(\mathrm{rad})\end{array}$ \\
\hline 1.2392 & 1.2394 & $2.0963 e-006$ & $1.9128 e-004$ \\
1.2915 & 1.2913 & $2.2918 e-006$ & $2.0000 e-004$ \\
1.3439 & 1.3436 & $2.4819 e-006$ & $6.5816 e-004$ \\
1.3963 & 1.3965 & $3.8608 e-006$ & $2.5959 e-004$ \\
1.4486 & 1.4493 & $2.1907 e-006$ & $1.9554 e-004$ \\
1.5010 & 1.5012 & $3.6759 e-006$ & $2.5329 e-004$ \\
1.5533 & 1.5535 & $2.3424 e-006$ & $2.0219 e-004$ \\
\hline
\end{tabular}

$16 \times K))$ plus the multiplications involved in crossover which is approximately $16 \times Q^{2}$ and the multiplication required for PS which is $16 \times K$. So, the total number of major multiplications involved in GA-PS for 2-L-shape array is $O\left(Q^{2}((3+32 \times K))+16 \times K\right)$. Similarly, the major computations
TABLE 12: Means, variances, and standard deviations at $10 \mathrm{~dB}$ noise for different elevation angles and fixed azimuth angle by using GAPS with 2-L-shape array.

\begin{tabular}{lccc}
\hline $\begin{array}{l}\theta \text { in radians for } \\
\phi=0.6109(\mathrm{rad})\end{array}$ & Mean of $\theta$ & Variance of $\theta$ & $\begin{array}{c}\text { Standard deviation } \\
\text { of } \theta\end{array}$ \\
\hline 1.2392 & 1.2392 & $2.6389 e-007$ & $6.7866 e-005$ \\
1.2915 & 1.2915 & $1.9565 e-007$ & $5.8436 e-005$ \\
1.3439 & 1.3439 & $4.0858 e-007$ & $8.4446 e-005$ \\
1.3963 & 1.3964 & $2.5674 e-007$ & $6.6940 e-005$ \\
1.4486 & 1.4487 & $2.3073 e-007$ & $6.3459 e-005$ \\
1.5010 & 1.5011 & $5.9533 e-007$ & $1.0193 e-004$ \\
1.5533 & 1.5534 & $2.0944 e-007$ & $6.0460 e-005$ \\
\hline
\end{tabular}

required for GA-PS with 1-L-shape array are $O\left(Q^{2}((3+20 \times\right.$ $K))+20 \times K)$ ), where $Q$ is the number of particles which is 12 in this work. The GA-PS technique with 2-L-shape array is less computationally expensive as compared to PM scheme with parallel-shape array [8]. However, it is little more computationally expensive from GA-PS with 1-L-shape array [15].

Case 9. In this simulation, we compared the RMSE of GAPS used for 2-L-shape array with its counterpart used for 1-L-shape array [15] as well as with PM for parallel-shape array [8]. In this, a single source is considered which has elevation and azimuth angles 1.0472(rad) and 1.9199(rad), respectively. The values of signal to noise ratio (SNR) are ranging from $5 \mathrm{~dB}$ to $25 \mathrm{~dB}$. For GA-PS with L-shape arrays, we have used $10 \log \times \sqrt{(\text { MSE) }}$ where MSE is defined in (16). It is quite obvious from Figure 6, that GA-PS with 2-L-shape array maintained minimum values of RMSE for all values of SNR. The second best RMSE is maintained by another GA-PS technique with 1-L-shape array. 
TABLE 13: Comparison among 2-L-shape aray, 1-L-shape array [15] and parallel-shape array [8].

\begin{tabular}{lccc}
\hline Property & Parallel-shape array [8] & 1-L-shape array [15] & 2-L-shape array \\
\hline Scheme used & PM & GA-PS & GA-PS \\
Elevation and azimuth angles range & $(0, \pi / 2),(0,2 \pi)$ & $(0, \pi / 2),(0,2 \pi)$ & $(0, \pi),(0,2 \pi)$ \\
Number of estimated sources & 2 & 2 & 2 \\
Number of sensors required & 33 & 7 & 4 \\
Number of Snapshots required & $M * T$ & Not required & Not required \\
Pair matching & Required & No failure & No failure \\
Failure estimation & $1.2217(\mathrm{rad})$ to $1.5708(\mathrm{rad})$ & &
\end{tabular}

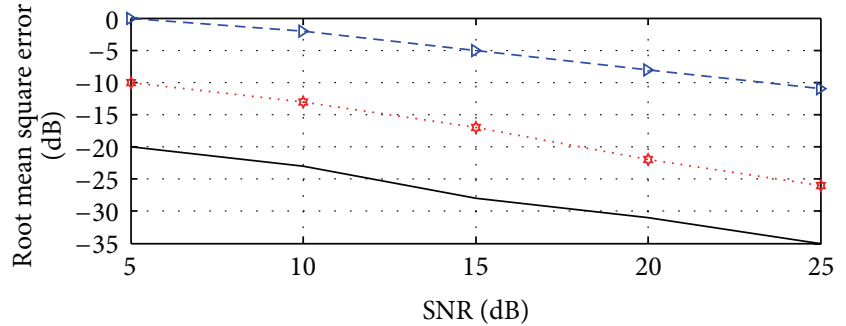

$\rightarrow-$ PM using parallel-shape array [8]

... GA-PS using 1-L-shape array [15]

_ GA-PS using 2-L-shape array

FIGURE 6: Root mean square error versus SNR.

Case 10. In Table 13, some general properties of parallelshape array [8], 1-L-shape array [15] and 2-L-shape array are listed. The main drawbacks of parallel-shape array with PM method include estimation failure in the range of 1.2217 ( $\mathrm{rad}$ ) to 1.5708 (rad), its computational complexity, pair matching problem, and need of more sensors. Some of the drawbacks are covered in 1-L-shape array by using GA-PS technique. However, the main disadvantage of 1-L-shape array [15] is the range limitation of elevation angles beyond $\pi / 2$, and it also requires more sensors. The 2-L-shape array with GA-PS technique is more effective and requires not only a minimum number of sensors but also covers the range of elevation angle beyond $\pi / 2$ to $\pi$ which is of great practical importance in mobile communication [21].

\section{Conclusion and Future Work Direction}

In this work, five techniques, that is, GA, PS, SA, SA-PS, and GA-PS, are used to jointly estimate amplitude, elevation angle and azimuth angle of far field sources impinging on 2L-shape array. It has been shown through various simulations that the hybrid GA-PS technique is the best technique among them. It was also found that the GA-PS technique used for 2-L shape array performed well in terms of estimation accuracy, convergence rate, RMSE, number of sensors required as compared to 1-L-shape array and PM for parallel-shape array. Hence, GA-PS approach with 2-L-shape array is more practical and less expensive in terms of hardware budget for wireless communications.

In future, one can look into same hybrid approach for null steering and sidelobe reduction as well.

\section{References}

[1] Z. U. Khan, A. Naveed, I. M. Qureshi, and F. Zaman, "Independent null steering by decoupling complex weights," IEICE Electronics Express, vol. 8, no. 13, pp. 1008-1013, 2011.

[2] M. A. Atique-ur-Rehman, F. zaman, I. M. Qureshi, and Y. A. Sheikh, "Null and sidelobes adjustment of damaged array using hybrid computing," in Proceedings of the IEEE International conference on Emerging Technology (ICET '12), pp. 386-389, Islamabad, Pakistan, 2012.

[3] H. Krim and M. Viberg, "Two decades of array signal processing research: the parametric approach," IEEE Signal Processing Magazine, vol. 13, no. 4, pp. 67-94, 1996.

[4] Q. I. Cheng, "Further study of the pencil-MUSIC algorithm," IEEE Transactions on Aerospace and Electronic Systems, vol. 32, no. 1, pp. 284-299, 1996.

[5] Y. Hua, T. K. Sarkar, and D. D. Weiner, "An L-shaped array for estimating 2-D directions of wave arrival," IEEE Transactions on Antennas and Propagation, vol. 39, no. 2, pp. 143-146, 1991.

[6] T. H. Liu and J. M. Mendel, "Azimuth and elevation direction finding using arbitrary array geometries," IEEE Transactions on Signal Processing, vol. 46, no. 7, pp. 2061-2065, 1998.

[7] V. S. Kedia and B. Chandna, "A new algorithm for 2-D DOA estimation," Signal Processing, vol. 60, no. 3, pp. 325-332, 1997.

[8] Y. Wu, G. Liao, and H. C. So, "A fast algorithm for 2-D directionof-arrival estimation," Signal Processing, vol. 83, no. 8, pp. 18271831, 2003.

[9] F. Zaman, I. M. Qureshi, A. Naveed, and Z. U. Khan, "Real time direction of arrival estimation in noisy environment using particle swarm optimization with single snapshot," Research Journal of Applied Sciences, Engineering and Technology, vol. 4, no. 13, pp. 1949-1952, 2012.

[10] Y. A. Sheikh, F. Zaman, I. M. Qureshi, and M. Atique-urRehman, "Amplitude and direction of arrival estimation using differential evolution," in IEEE conference on Emerging Technology (ICET), pp. 386-389, Islamabad, Pakistan, 2012.

[11] A. K. Junaid, M. A. Z. Raja, and I. M. Qureshi, "Stochastic computational approach for complex non-linear ordinary differential equations," Chinese Physics Letters, vol. 28, Article ID 020206, 2011.

[12] A. K. Junaid, M. A. Z. Raja I, and M. Qureshi, "Numerical treatment of nonlinear Emden-Fowler equation using Stochastic Technique," Annals of Mathematics and Artificial Intelligence, vol. 63, no. 2, pp. 185-207, 2011.

[13] F. Zaman, I. M. Qureshi, A. Naveed, and Z. U. Khan, "Joint estimation of amplitude, direction of arrival and range of near field sources using memetic computing," Progress in Electromagnetics Research C, vol. 31, pp. 199-213, 2012. 
[14] F. Zaman, J. A. Khan, Z. U. Khan, and I. M. Qureshi, "An application of hybrid computing to estimate jointly the amplitude and direction of arrival with single snapshot," in Proceedings of the IEEE 10th IBCAST Conference, Islamabad, Pakistan, 2012.

[15] F. Zaman, I. M. Qureshi, A. Naveed, J. A. Khan, and R. M. A. Zahoor, "Amplitude and directional of arrival estimation: comparison between different techniques," Progress in Electromagnetics Research B, vol. 39, pp. 319-335, 2012.

[16] F. Harabi, H. Changuel, and A. Gharsallah, "Direction of arrival estimation method using A 2-L shape arrays antenna," Progress in Electromagnetics Research, vol. 69, pp. 145-160, 2007.

[17] J. De Vicente, J. Lanchares, and R. Hermida, "Placement by thermodynamic simulated annealing," Physics Letters A, vol. 317, no. 5-6, pp. 415-423, 2003.

[18] X. Zhang and J. Ma, "Pattern search methods for finite minimax problems," Journal of Applied Mathematics and Computing, vol. 32, pp. 491-506, 2010.

[19] L. Zhang, Y.-C. Jiao, B. Chen, and H. Li, "Orthogonal genetic algorithm for planar thinned array designs," International Journal of Antennas and Propagation, vol. 2012, Article ID 319037, 7 pages, 2012.

[20] H. Gargama, S. K. Chaturvedi, and A. K. Thakur, "On the design and reliability analysis of electromagnetic absorbers using realcoded genetic algorithm and monte carlo simulation," Progress in Electromagnetics Research B, vol. 43, pp. 169-187, 2012.

[21] N. A. H. M. Tayem, Direction of arrival angle estimation schemes for wirelerss communication systems [Ph.D. thesis], Wichita State University, 2005. 

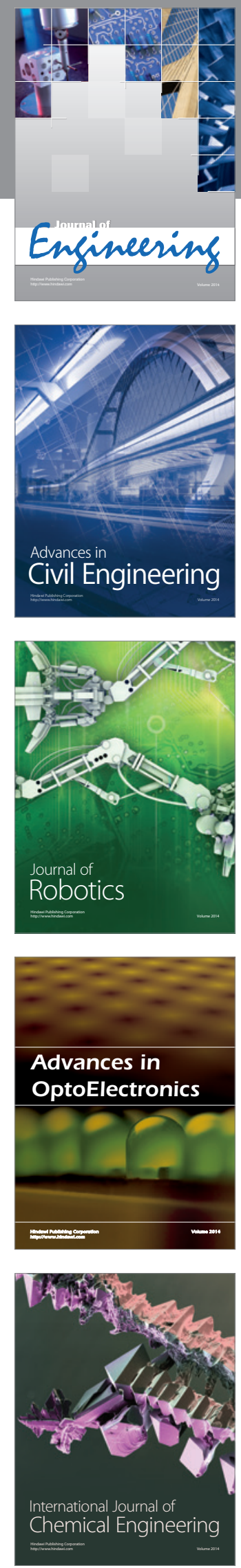

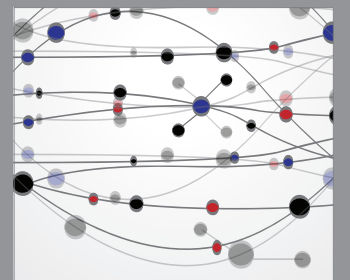

The Scientific World Journal
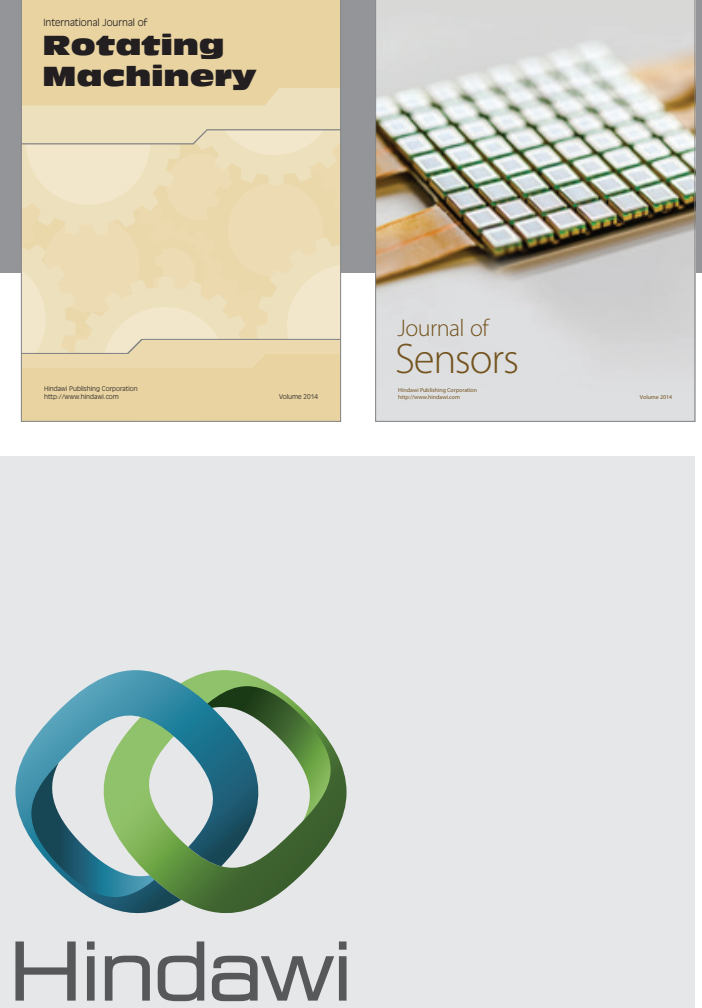

Submit your manuscripts at http://www.hindawi.com
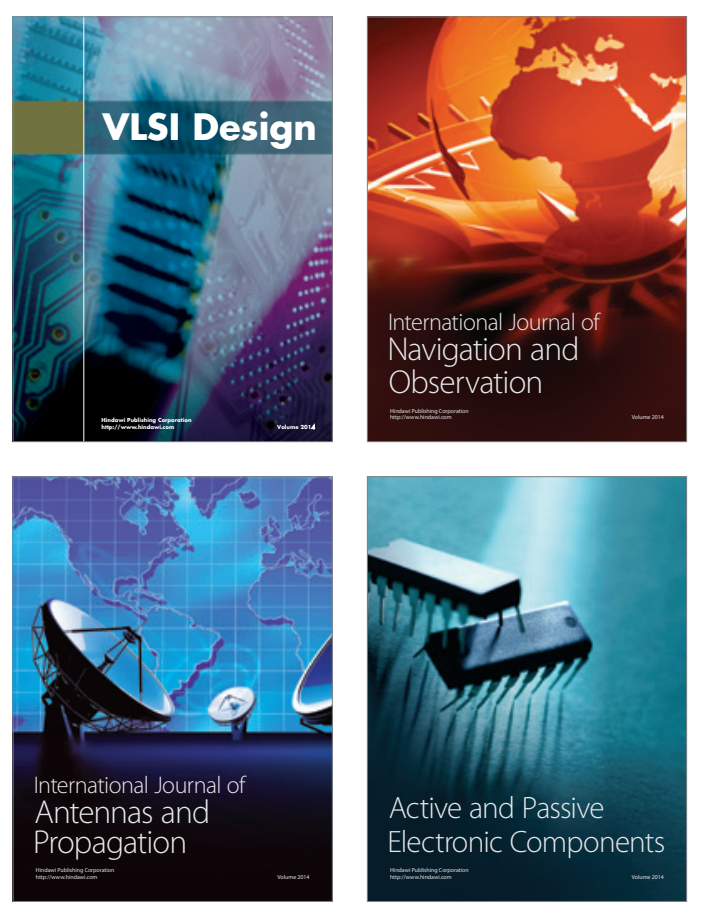
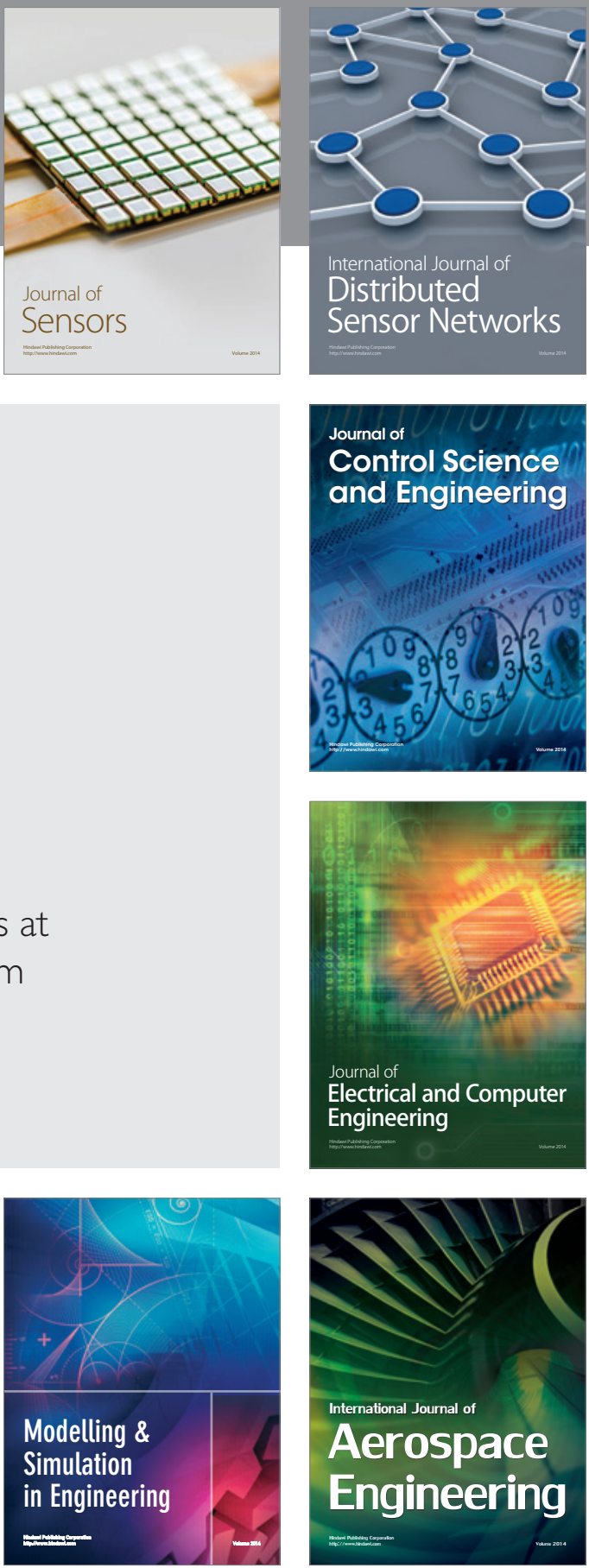

Journal of

Control Science

and Engineering
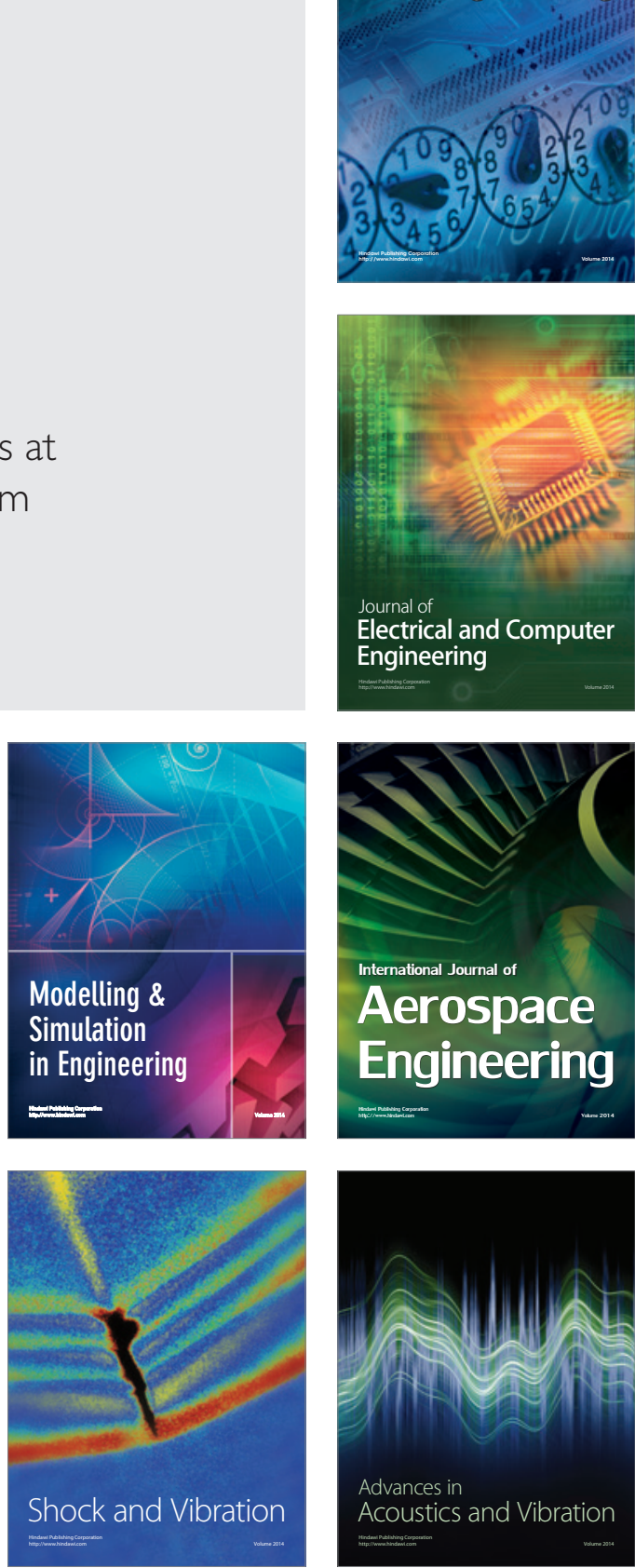